OPEN ACCESS

Edited by:

Di Lang,

University of Wisconsin-Madison,

United States

Reviewed by:

Marina Cerrone,

New York University, United States

Masafumi Takahashi,

Jichi Medical University, Japan

*Correspondence:

$\mathrm{Na} \mathrm{Li}$

nal@bcm.edu

Specialty section:

This article was submitted to

Cardiac Electrophysiology,

a section of the journal

Frontiers in Physiology

Received: 17 May 2018

Accepted: 25 July 2018

Published: 13 August 2018

Citation:

Chen G, Chelu MG, Dobrev D and

Li N (2018) Cardiomyocyte

Inflammasome Signaling

in Cardiomyopathies and Atrial Fibrillation: Mechanisms and Potential

Therapeutic Implications.

Front. Physiol. 9:1115

doi: 10.3389/fphys.2018.01115

\section{Cardiomyocyte Inflammasome Signaling in Cardiomyopathies and Atrial Fibrillation: Mechanisms and Potential Therapeutic Implications}

\author{
Gong Chen ${ }^{1}$, Mihail G. Chelu ${ }^{2,3}$, Dobromir Dobrev ${ }^{4}$ and $\mathrm{Na} \mathrm{Li}^{1,5,6 *}$ \\ ${ }^{1}$ Section of Cardiovascular Research, Department of Medicine, Baylor College of Medicine, Houston, TX, United States, \\ ${ }^{2}$ Comprehensive Arrhythmia Research and Management Center, School of Medicine, University of Utah, Salt Lake City, UT, \\ United States, ${ }^{3}$ Cardiovascular Medicine Division, Section of Cardiac Electrophysiology, School of Medicine, University \\ of Utah, Salt Lake City, UT, United States, ${ }^{4}$ Institute of Pharmacology, West German Heart and Vascular Center, University \\ Duisburg-Essen, Essen, Germany, ${ }^{5}$ Cardiovascular Research Institute, Baylor College of Medicine, Houston, TX, \\ United States, ${ }^{6}$ Department of Molecular Physiology and Biophysics, Baylor College of Medicine, Houston, TX, \\ United States
}

Inflammasomes are high molecular weight protein complexes in the cytosol of immune and other cells that play a critical role in the innate immune system in response to cellular stress. NLRP3 inflammasome, the best-understood inflammasome, is known to mediate the maturation (activation) of caspase-1 from pro-caspase-1, causing the maturation and release of cytokines (e.g., interleukin-1 $\beta$ ) and potentially leading to a form of inflammatory programmed cell death called pyroptosis. Previous work has shown that the NLRP3 components are expressed in cardiomyocytes and cardiac fibroblasts and recent studies have identified the NLRP3 inflammasome as a key nodal point in the pathogenesis of cardiomyopathies and atrial fibrillation, which may create an opportunity for the development of new therapeutic agents. Here we review the recent evidence for a role of NLRP3 inflammasome in the cardiomyocytes and discuss its potential role in the evolution of cardiac remodeling and arrhythmias and new opportunities created by these very recent developments.

Keywords: NLRP3 inflammasome, innate immune system, cardiomyocytes, cardiac fibroblasts, cardiomyopathy, atrial fibrillation

\section{THE INNATE IMMUNE SYSTEM, INFLAMMATION AND INFLAMMASOME SIGNALING}

Inflammation is a vital biological process involving an acute response to infection and tissue damage aiming to prevent harmful influence to the host (Medzhitov, 2008; Buckley et al., 2013). The mammalian innate immune system plays an important role in recognizing foreign pathogenor damage-associated molecular patterns (PAMPs and DAMPs, respectively) and defending the host against infection or injury caused by other pathological organisms (Matzinger, 1994).

Inflammasome acts as an intracellular innate immune sensor (Martinon et al., 2002). The inflammasome is a multi-protein signaling platform that generally involves 3 proteins: (1) a NODlike receptor (NLR), (2) an adaptor protein like apoptosis-associated speck-like protein containing a CARD (ASC), and (3) a cysteine protease such as caspase-1 or caspase-5 (Kanneganti, 2015). 
NLRs are a class of pattern recognition receptors (PRRs) that act as a sensor for the inflammasome. At least 22 different NLR proteins have been identified in humans and 34 in mouse (Ting et al., 2008). Most NLRs consist of a tripartite structure that includes: (1) a N-terminal caspase-recruitment domain (CARD) or pyrin domain that mediates downstream protein-protein assembly, (2) a centrally located nucleotide-binding-and-oligomerization domain that facilitates self-oligomerization, and (3) a C-terminal leucine-rich repeats (LRRs) that are thought to be involved in stimuli sensing (Martinon et al., 2002). To date, the best investigated and validated inflammasome type is the "NACHT, LRR and PYD domain containing protein 3" (NLRP3) inflammasome (He et al., 2016). Upon recognizing a series of inflammation-inducing stimuli (e.g., PAMPs and DAMPs), NLRP3 inflammasomes in the innate immune cells activate caspase-1 (Casp-1) which promotes the production of proinflammatory cytokines (IL-1 $\beta$ and pro-IL-18) and may lead to cell death known as pyroptosis (Schroder and Tschopp, 2010; Davis et al., 2011). The activation of the NLRP3 inflammasome requires two primary signals (Figure 1): (1) a priming step in which the toll-like receptor (TLR)-nuclear factor- $\kappa \mathrm{B}(\mathrm{NF \kappa} \mathrm{B})$ signaling pathway promotes the transcription of NLRP3 and precursor ILs (pro-IL1 $\beta$ or pro-IL-18); and (2) a triggering step in which a series of stimuli $\left(\mathrm{K}^{+}\right.$efflux, increase in cytosolic $\mathrm{Ca}^{2+}$, generation of reactive oxygen species [ROS], mitochondrial dysfunction, and lysosomal rupture) (He et al., 2016) can facilitate the assembly of the NLRP3 inflammasome components by recruiting precursor-caspase-1 (pro-Casp-1) into the complex via the adaptor protein ASC. This promotes the autocleavage of pro-Casp-1 to Casp-1 containing the active p20/p10 heterodimer proteins (Wilson et al., 1994). Alternatively, NLRP3 inflammasome may also activate caspase-11 or human orthologues caspase-4/caspase-5 (casp-4/5), which is also known as "non-canonical" NLRP3 inflammasome pathway (Kanneganti, 2015). Activated Casp1 holoenzyme further cleaves pro-IL-1 $\beta$ and pro-IL-18 to form their respective mature forms ( $\mathrm{Li}$ et al., 1995; Gu et al., 1997). Mature IL-1 $\beta$ is a potent proinflammatory mediator in many immune reactions, including the recruitment of innate immune cells to the site of infection and modulation of adaptive immune cells, whereas mature IL-18 is important for the production of interferon- $\gamma$ and potentiation of cytotoxic activity of natural killer and $\mathrm{T}$ cells (Dinarello, 2009).

In addition, active Casp-1 may promote pyroptosis, which is characterized by increased membrane permeability with extracellular release of pro-inflammatory signaling molecules and cell swelling and eventually cell membrane rupture (Fink et al., 2008; Toldo and Abbate, 2018). Oligomerization of N-terminal fragments resulting from the cleavage of gasdermin D by Casp1 lead to formation of membrane pores, which are permeable for mature IL-1 $\beta$, IL-18, and active Casp-1 (Liu et al., 2016). Furthermore, cleavage of several proteins involved in the Krebs cycle by Casp-1 leads to a significant decrease in cell energy production which results in cell swelling and rupture (Shao et al., 2007).

\section{NLRP3 INFLAMMASOME SIGNALING IN THE HEART}

Cardiomyocytes (CMs) make up approximately 75\% of normal adult myocardial tissue (Camelliti et al., 2005). Although non-cardiomyocytes occupy a relatively small fraction of myocardial volume, they are essential for normal cardiac function by providing extracellular matrix (ECM), intercellular communication, and vascular supply needed for efficient function and survival of CMs (Travers et al., 2016). The NLRP3 inflammasome components have been identified in both CMs and cardiac fibroblasts (CFs), which are the two most abundant cell populations in the mammalian heart (Baudino et al., 2006). An augmented function of NLRP3 inflammasome has been proposed to play a role in multiple human diseases, such as auto-inflammatory disease (Hoffman and Wanderer, 2010), diabetes (Vandanmagsar et al., 2011), atherosclerosis (Duewell et al., 2010; Baldrighi et al., 2017), and ischemic cardiomyopathy (Kawaguchi et al., 2011; Mezzaroma et al., 2011; Sandanger et al., 2013; Liu et al., 2014; Toldo et al., 2016). The innate immune system functions as the primary cardiac defense against pathogens and tissue damage (Askevold et al., 2014). Myocardial infarction is the most common cause of cardiac injury (Jennings et al., 1990), resulting from coronary atherosclerosis-mediated plaque rupture and involving acute loss of CMs. Necrotic cardiac cells can trigger inflammatory cascades to get rid of dead cell debris in the infarcted area (Pfeffer and Braunwald, 1990; Opie et al., 2006). Conversely, cell death can also release intracellular components, which further stimulate innate immune mechanisms to facilitate the inflammatory responses. Endogenous ligands released after injury can be recognized as danger signals by cell surface receptors, thereby activating cellular inflammatory signaling (Beg, 2002). TLR-mediated pathways can trigger post-infarction inflammatory responses by activating toll-like receptor (TLR)nuclear factor-kB (NFkB) and related signaling (Lawrence, 2009). Chemokines recruit inflammatory leukocytes to the infarcted area, and cytokines promote leukocyte-endothelial cell adhesions. Moreover, transforming growth factor- $\beta$ (TGF- $\beta$ ) and interleukin-10 (IL-10) can promote cardiac repair by suppressing inflammation, enhancing fibroblast-to-myofibroblast transition, and promoting ECM deposition (Kaur et al., 2009; Frangogiannis, 2014). Compared to the well-established canonical function of the NLRP3 inflammasome in the innate immune cells, the putative role of the NLRP3 inflammasome in non-immune cells including cardiac cells is poorly defined. NLRP3 and other important components of the inflammasome may not be constitutively expressed in healthy mouse and human heart but expression is induced in leukocytes, endothelial cells, CFs in the granulation tissue and CMs in the infarct border zones in a mouse model of acute MI (Yin et al., 2009; Mezzaroma et al., 2011). NLRP3, IL-1 $\beta$, and IL-18 mRNA levels were shown to be increased in both left ventricle CMs and CFs in a post-MI mouse model (Sandanger et al., 2013). ASC is constitutively expressed in mouse CMs and CFs (Kawaguchi et al., 2011). The entire signaling cascade appears to be operative in CFs: NLRP3 inflammasome activation by DAMP molecule 


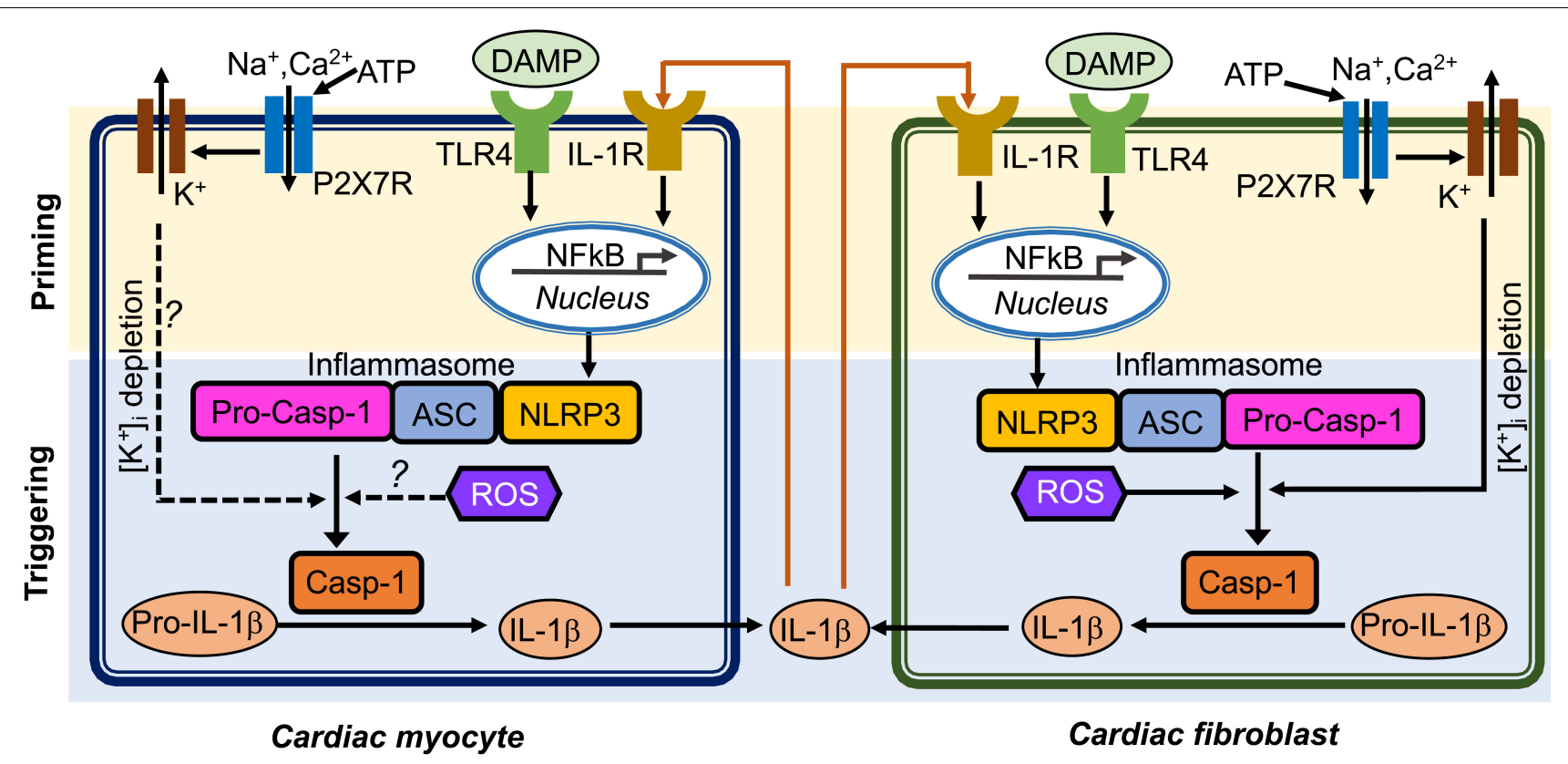

FIGURE 1 | Putative mechanisms of NLRP3 inflammasome activation in cardiomyocytes and cardiac fibroblasts. Question marks indicated the mechanisms that need to be investigated. ASC, apoptosis-associated speck-like protein containing a CARD; ATP, adenosine triphosphate; Casp-1, caspase-1; DAMP, damage-associate molecular pattern; IL-1 $\beta$, interleukin-1 $\beta$; IL-1R, interleukin-1 receptor; NFKB, nuclear factor kappa-light-chain-enhancer of activated B cells; NLRP3, NACHT, LRR and PYD domain containing protein 3; ROS, reactive oxidative species; pro-Casp-1, precursor Caspase-1; P2X7R, P2X7 receptor; TLR4, toll-like receptor 4.

ATP, TLR ligand specific activation in a NFKB dependent manner, assembly of the NLRP3/ASC inflammasome, and activation of Casp-1 (Kawaguchi et al., 2011; Sandanger et al., 2013). ASC was highly expressed in the inflammatory infiltrate cells and weakly expressed in CMs and the interstitial cells obtained from patients who had died after an acute myocardial infarction (Kawaguchi et al., 2011). Figure 1 illustrates the putative mechanisms potentially underlying the activation of the NLRP3 inflammasome and the postulated interaction patterns between CMs and CFs through complex autocrine and paracrine mechanisms (Figure 1). In this review, we will focus on the very recently established role of the NLRP3 inflammasome in cardiac cells and its potential involvement in cardiac diseases, such as cardiomyopathies and atrial fibrillation (AF).

\section{ROLE OF NLRP3 INFLAMMASOME IN CARDIOMYOPATHIES}

Cardiomyopathies are cardiac diseases that severely impact patient morbidity and mortality (Wexler et al., 2009). They can be induced by myocardial injury that is often accompanied by transient or persistent local inflammatory responses. This type of inflammation is deemed as sterile inflammation due to the lack of a microbial pathogen. A number of studies have illustrated a central role of NLRP3 inflammasome in murine models of ischemic and non-ischemic cardiomyopathies (Mezzaroma et al., 2011; Bracey et al., 2013; Liu et al., 2014; Toldo et al., 2016; Valle Raleigh et al., 2017). NLRP3 inflammasome can be activated by several signals generated during the initial ischemia-induced myocardial injury: dsDNA, RNA, and ATP released from dying cells. DAMPs including dsDNA and RNA can activate the TLR-NFkB signaling pathway and promote the "priming" of NLRP3 and pro-IL1 $\beta$. On the other hand, ATP can activate P2X purinoceptor 7 (P2X7R) in CMs thereby enhancing the $\mathrm{K}^{+}$efflux and subsequently facilitating the assembly of NLRP3 inflammasome complex, which promotes the autocatalytic activation of Casp-1. The mature Casp-1 further perpetuates myocardial remodeling via two established mechanisms. On one hand, mature Casp-1 can increase the production of IL- $1 \beta$ and IL-18 by cleaving their precursor proteins into the mature forms. An increased release of IL-1 $\beta$ and IL-18 will spread and amplify the local inflammation and promote fibrosis, a major factor contributing to the structural remodeling of myocardium (Nguyen et al., 2017). On the other hand, mature Casp-1 cleaves gasdermin-D (GSDMD), another crucial component of the NLRP3 inflammasome, resulting in the formation of the N-terminal proteolytic fragment of GSDMD (GSDMD$\mathrm{Nt}$ ), which can promote inflammatory cell death known as "pyroptosis" (He et al., 2015; Shi et al., 2015), further deteriorating the function of the remaining myocardium. Moreover, GSDMD-Nt is not only a potential executor of pyroptosis, but is also a requirement for the release of IL-1 $\beta$, because genetic deletion of GSDMD precluding the GSDMD membrane pore formation eliminates the ability of the cells to release IL-1 $\beta$ (He et al., 2015; Sborgi et al., 2016). Although it is unclear which degree of NLRP3 inflammasome 
activation is associated with pyroptosis induction, Mezzaroma et al. (2011) has demonstrated that the Casp-1-mediated cell death is restricted to the granulation tissue and CMs located to the infarct border zone following acute myocardium infarction.

The NLRP3 inflammasome might also be implicated in the pathogenesis of non-ischemic cardiomyopathy. Bracey et al. have shown that the development of cardiac hypertrophy, apoptosis and ventricular dilatation in the cardiac-specific calcineurin transgenic mice (CN-Tg) (Bracey et al., 2013) is associated with increased protein levels of NLRP3 in cardiac tissue and IL-1 $\beta$ in serum, which suggest that both the "priming" and "triggering" steps are activated during the development of heart failure with reduced ejection fraction (HFrEF). Most importantly, administration of IL-1 receptor antagonist (IL-1ra) prevented the progressive reduction of cardiac contractility, reduced infiltration of inflammatory cells in the myocardium, and decreased apoptosis in $\mathrm{CN}$-Tg mice.

The pathogenesis of diabetic cardiomyopathy has also been linked to the activation of NLRP3 inflammasome (Shaw et al., 2010). Diabetic cardiomyopathy often manifests as heart failure with preserved ejection fraction (HFpEF), and is a consequence of increased ventricular wall stiffness leading to left ventricular diastolic dysfunction. It is well established that hyperglycemia increases the production of ROS, which is a known trigger for the assembly of the NLRP3 inflammasome complex. Glucose itself has also been reported to be an effective activator of the NLRP3 inflammasome (Shi et al., 2015; $\mathrm{Zu}$ et al., 2015). In a type-2 diabetic rat model induced by high fat diet and low dose streptozotocin, the expression of NLRP3, ASC, Casp-1, and IL-1 $\beta$ was increased in the heart. Genetic inhibition of Nlrp3 by a small interfering RNA in vivo improved left ventricular diastolic function in these diabetic rats (Luo et al., 2014), which was attributed to a reduction in cell death, an improvement of myofilament and mitochondria structures, and a reduction in cardiac fibrosis. Thus there is accumulating evidence pointing to a critical role of NLRP3 inflammasome activation in ischemic and non-ischemic cardiomyopathy.

\section{ROLE OF NLRP3 INFLAMMASOME IN AF}

$\mathrm{AF}$ is the most frequent clinical arrhythmia which is associated with an increased risk of stroke and worsening heart failure (Andrade et al., 2014; Freeman et al., 2017). The development of AF often involves ectopic triggers acting on an arrhythmogenic substrate to initiate AF-maintaining reentry (Heijman et al., 2014). The current therapeutic approaches are moderately effective perhaps because of critical gaps in current knowledge about arrhythmia mechanisms and important translational challenges of available therapeutic concepts (Heijman et al., 2016, 2018).

An enhanced inflammatory response is frequently associated with AF development (Aviles et al., 2003; Harada et al., 2015) and increased levels of circulating IL-1 $\beta$ and IL-18 positively correlated with progression from paroxysmal AF (pAF) to long-lasting persistent AF (perAF), along with left atrial dilatation (an independent risk factor of AF) in AF patients (Luan et al., 2010; Gungor et al., 2013). We recently showed that the activity of the NLRP3 inflammasome is increased in CMs from patients with pAF and perAF (Yao et al., 2018). In atrial CMs from pAF patients, protein levels of active Casp-1p20 were significantly increased, despite the unchanged protein levels of NLRP3 and pro-Casp-1, likely reflecting the fact that the increase in NLRP3 inflammasome activity in pAF might be due to an increased "triggering" (enhanced assembly), rather than "priming" (increased gene transcription) processes. In contrast, atrial CMs from perAF patients showed not only higher protein levels of Casp-1-p20, but also an upregulation of NLRP3, ASC, and pro-Casp-1 proteins, indicating that both "priming" and "triggering" processes contribute to the activation of the NLRP3 inflammasome in CMs of these patients. To the best of our knowledge this study is the first to show that the NLRP3 inflammasome is expressed and upregulated in non-immune cardiac cells (CMs) from pAF and perAF patients and that its activity in human CMs correlates with the progression of AF to more persistent forms.

To determine whether CM-restricted activation of the NLRP3 inflammasome plays a causative role in AF pathogenesis, a CM-specific knockin mouse model expressing a gain-offunction mutation of NLRP3 (NLRP3 ${ }^{\mathrm{A} 350 \mathrm{~V}}$ ) mimicking the constitutive NLRP3 activation seen in CMs from AF patients, was established by crossing a previously established conditional allele (Brydges et al., 2009) to the CM-specific Cre transgenic mouse $\left(M y h 6^{C r e}: N l r p 3^{A 350 V /+}, \mathrm{CM}-\mathrm{KI}\right)$. In this CM-KI mouse model, total protein levels of NLRP3, ASC and pro-Casp1 remained unchanged, whereas Casp-1-p20 protein levels were increased, recapitulating the changes we observed in pAF patients. Electrophysiological studies have demonstrated that the constitutive activation of NLRP3 inflammasome in CMs only increased the AF susceptibility by producing both ectopic (triggered) activity and reentry-promoting electrical remodeling in CM-KI mice (Yao et al., 2018). Moreover, the enhanced AF susceptibility was associated with abnormal diastolic sarcoplasmic reticulum (SR) $\mathrm{Ca}^{2+}$ releases due to increased protein levels of ryanodine receptor type-2 (RyR2), which might represent the molecular correlates of ectopic activity as reflected by the higher incidence of premature atrial contractions. In addition, the atrial effective refractory period (AERP) was abbreviated most likely because of an enhanced function of the ultra-rapid delayed-rectifier $\mathrm{K}^{+}$current (Kv1.5) in CMs. Genetic inhibition of Nlrp3 in CMKI mice using the adeno-associated virus type 9 (AAV9)mediated gene transfer of a short-hairpin RNA (shRNA), reduced the incidence of inducible AF episodes. Thus, this study clearly validated the causal relationship between the CMspecific NLRP3 inflammasome activation and the susceptibility to AF (Yao et al., 2018). Figure 2 summarizes the putative molecular mechanisms associated with AF development due to the activation of the NLRP3 inflammasome in CMs only. Since NLRP3 inflammasomes exist also in CFs and CFs play an important role in atrial fibrosis, a well-recognized substrate for AF maintenance, future studies should address 


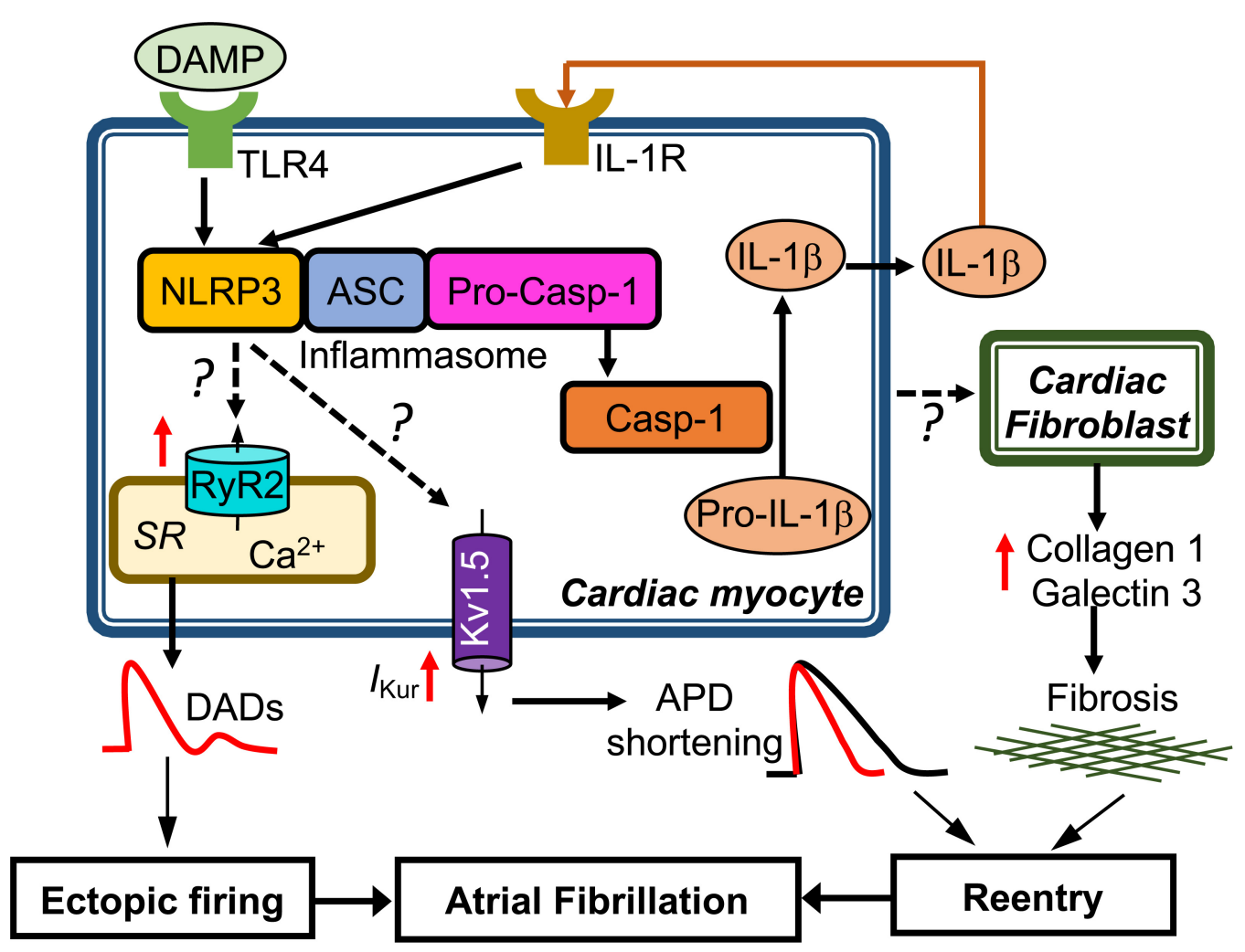

FIGURE 2 | Putative molecular mechanisms underlying the development of atrial fibrillation due to constitutive activation of NLRP3 inflammasome in mouse cardiac myocytes only. Question marks indicated the mechanisms that need to be investigated. APD, action potential duration; DADs, delayed afterdepolarizations; IKur, ultra-rapid delayed rectifier potassium current; SR, sarcoplasmic reticulum.

the potential role of the CF NLRP3 inflammasome for AF pathophysiology.

\section{TARGETING OF THE NLRP3 INFLAMMASOME AS A THERAPEUTIC OPTION}

Based on available experimental evidence, targeting the NLRP3 inflammasome-signaling pathway may represent a unique therapeutic opportunity for patients with cardiomyopathy and/or AF. Most of the agents described below have been tested only pre-clinically in animal models and their potential translation into clinical practice will require prospective clinical trials in the suitable patient populations. The multiple players involved in the NLRP3 inflammasome signaling cascade offer a variety of viable options for therapeutic exploitation. Targeting the upstream regulators of NLRP3 inflammasome function, selective inhibition of different members of NLRP3 inflammasome complex, interruption of the complex preventing maturation of Casp1 , and selective blockade of the downstream effectors (mature caspase- 1 and IL-1 $\beta$ ) of NLRP3-inflammasome activation, may all be viable therapeutic interventions. An inhibition of the upstream regulators could be achieved by inhibition of $\mathrm{K}^{+}$ efflux with the antidiabetic drug glyburide (Haque et al., 2016) or the application of a ROS-scavenger (Liu et al., 2014). The inhibition of individual NLRP3 inflammasome members could be accomplished by genetic silencing of Nlrp3, ASC, or pro-Casp-1 using specific shRNAs (Dai et al., 2011; Yao et al., 2018) or CRISP/Cas9-mediated non-homologous end joining (NHEJ) (Zhang et al., 2014) via the AAV-mediated gene transfer system. Disruption of inter-domain interaction between NLRP3 and ASC can be achieved using the MCC950 compound (Coll et al., 2015). The blockade of IL-1 $\beta$ functions is currently the most advanced strategy with two agents being clinically available: the IL-1 receptor antagonist anakinra (Mitroulis et al., 2010) and the neutralizing IL-1 $\beta$ antibody canakinumab (Jesus and Goldbach-Mansky, 2014; Ridker et al., 2017). Moreover, several Casp-1 inhibitors (e.g., Ac-WEHDCho and Ac-YVAD-cho) are currently under development for patients with auto-inflammatory diseases (Howley and Fearnhead, 2008; MacKenzie et al., 2010). Interestingly, the recently completed large-scale clinical trial "The Canakinumab Anti-inflammatory Thrombosis Outcomes Study” (CANTOS) showed that selective targeting of the IL-1 $\beta$ pathway can significantly reduce the rate of recurrent cardiovascular events including myocardial infarction, and that this effect was independent of lipid levels lowering (Ridker et al., 2017). This study provides a promise that targeting of the NLRP3inflammasome could potentially be a viable therapeutic option 
for cardiomyopathies, AF, and perhaps other cardiovascular diseases including heart failure. Prospective randomized clinical trials including suitable clinical patient populations are needed to prove and validate the therapeutic potential of NLRP3 inflammasome inhibition for the management of cardiovascular diseases.

\section{AUTHOR CONTRIBUTIONS}

NL designed the study. GC organized the database. GC and MC wrote sections of the manuscript. DD and NL revised the manuscript critically for important

\section{REFERENCES}

Andrade, J., Khairy, P., Dobrev, D., and Nattel, S. (2014). The clinical profile and pathophysiology of atrial fibrillation: relationships among clinical features, epidemiology, and mechanisms. Circ. Res. 114, 1453-1468. doi: 10.1161/ CIRCRESAHA.114.303211

Askevold, E. T., Gullestad, L., Dahl, C. P., Yndestad, A., Ueland, T., and Aukrust, P. (2014). Interleukin-6 signaling, soluble glycoprotein 130, and inflammation in heart failure. Curr. Heart Fail. Rep. 11, 146-155. doi: 10.1007/s11897-0140185-9

Aviles, R. J., Martin, D. O., Apperson-Hansen, C., Houghtaling, P. L., Rautaharju, P., Kronmal, R. A., et al. (2003). Inflammation as a risk factor for atrial fibrillation. Circulation 108, 3006-3010. doi: 10.1161/01.CIR.0000103131. 70301.4F

Baldrighi, M., Mallat, Z., and Li, X. (2017). NLRP3 inflammasome pathways in atherosclerosis. Atherosclerosis 267, 127-138. doi: 10.1016/j.atherosclerosis. 2017.10.027

Baudino, T. A., Carver, W., Giles, W., and Borg, T. K. (2006). Cardiac fibroblasts: friend or foe? Am. J. Physiol. Heart Circ. Physiol. 291, H1015-H1026. doi: 10.1152/ajpheart.00023.2006

Beg, A. A. (2002). Endogenous ligands of Toll-like receptors: implications for regulating inflammatory and immune responses. Trends Immunol. 23, 509-512. doi: 10.1016/S1471-4906(02)02317-7

Bracey, N. A., Beck, P. L., Muruve, D. A., Hirota, S. A., Guo, J., Jabagi, H., et al. (2013). The Nlrp3 inflammasome promotes myocardial dysfunction in structural cardiomyopathy through interleukin-1ß. Exp. Physiol. 98, 462-473. doi: 10.1113/expphysiol.2012. 068338

Brydges, S. D., Mueller, J. L., McGeough, M. D., Pena, C. A., Misaghi, A., Gandhi, C., et al. (2009). Inflammasome-mediated disease animal models reveal roles for innate but not adaptive immunity. Immunity 30, 875-887. doi: 10 . 1016/j.immuni.2009.05.005

Buckley, C. D., Gilroy, D. W., Serhan, C. N., Stockinger, B., and Tak, P. P. (2013). The resolution of inflammation. Nat. Rev. Immunol. 13, 59-66. doi: 10.1038/ nri3362

Camelliti, P. L., Borg, T. K., and Kohl, P. (2005). Structural and functional characterisation of cardiac fibroblasts. Cardiovasc. Res. 1, 40-51. doi: 10.1016/j. cardiores.2004.08.020

Coll, R. C., Robertson, A. A., Chae, J. J., Higgins, S. C., Muñoz-Planillo, R., Inserra, M. C., et al. (2015). A small-molecule inhibitor of the NLRP3 inflammasome for the treatment of inflammatory diseases. Nat. Med. 21, 248-255. doi: 10.1038/ nm.3806

Dai, X., Sayama, K., Tohyama, M., Shirakata, Y., Hanakawa, Y., Tokumaru, S., et al. (2011). Mite allergen is a danger signal for the skin via activation of inflammasome in keratinocytes. J. Allergy Clin. Immunol. 127, 806-814. doi: 10.1016/j.jaci.2010.12.006

Davis, B. K., Wen, H., and Ting, J. P. (2011). The inflammasome NLRs in immunity, inflammation, and associated diseases. Аnnu. Rev. Immunol. 29, 707-735. doi: 10.1146/annurev-immunol-031210101405 intellectual content. All authors contributed to the manuscript revision, read, and approved the submitted version.

\section{FUNDING}

This work was supported by the National Institutes of Health (R56-HL131649 to NL, R01-HL136389 to NL, and DD, R01-HL131517 to DD), the American Heart Association (14SDG20080008 to NL), the German Research Foundation DFG (Do 769/4-1 to DD), and DZHK (German Center for Cardiovascular Research to DD).

Dinarello, C. A. (2009). Immunological and inflammatory functions of the interleukin-1 family. Annu. Rev. Immunol. 27, 519-550. doi: 10.1146/annurev. immunol.021908.132612

Duewell, P., Kono, H., Rayner, K. J., Sirois, C. M., Vladimer, G., Bauernfeind, F. G., et al. (2010). NLRP3 inflammasomes are required for atherogenesis and activated by cholesterol crystals. Nature 464, 1357-1361. doi: 10.1038/ nature 08938

Fink, S. L., Bergsbaken, T., and Cookson, B. T. (2008). Anthrax lethal toxin and Salmonella elicit the common cell death pathway of caspase-1-dependent pyroptosis via distinct mechanisms. Proc. Natl. Acad. Sci. U.S.A. 105, 43124317. doi: $10.1073 /$ pnas.0707370105

Frangogiannis, N. G. (2014). Targeting the transforming growth factor (TGF)- $\beta$ cascade in the remodeling heart: benefits and perils. J. Mol. Cell. Cardiol. 76, 169-171. doi: 10.1016/j.yjmcc.2014.09.001

Freeman, J. V., Wang, Y., Akar, J., Desai, N., and Krumholz, H. (2017). National trends in atrial fibrillation hospitalization, readmission, and mortality for medicare beneficiaries, 1999-2013. Circulation 135, 1227-1239. doi: 10.1161/ CIRCULATIONAHA.116.022388

Gu, Y., Kuida, K., Tsutsui, H., Ku, G., Hsiao, K., Fleming, M. A., et al. (1997). Activation of interferon-gamma inducing factor mediated by interleukin-1beta converting enzyme. Science 275, 206-209. doi: 10.1126/science.275.5297.206

Gungor, B., Ekmekci, A., Arman, A., Ozcan, K. S., Ucer, E., Alper, A. T., et al. (2013). Assessment of interleukin-1 gene cluster polymorphisms in lone atrial fibrillation: new insight into the role of inflammation in atrial fibrillation. Pacing Clin. Electrophysiol. 36, 1220-1227. doi: 10.1111/pace.12182

Haque, S., Lan, X., Wen, H., Lederman, R., Chawla, A., Attia, M., et al. (2016). HIV promotes nlrp3 inflammasome complex activation in murine hiv-associated nephropathy. Am. J. Pathol. 186, 347-358. doi: 10.1016/j.ajpath.2015.10.002

Harada, M., Van Wagoner, D. R., and Nattel, S. (2015). Role of inflammation in atrial fibrillation pathophysiology and management. Circ. J. 79, 495-502. doi: 10.1253/circj.CJ-15-0138

He, W. T., Wan, H., Hu, L., Chen, P., Wang, X., Huang, Z., et al. (2015). Gasdermin $\mathrm{D}$ is an executor of pyroptosis and required for interleukin-1beta secretion. Cell Res. 25, 1285-1298. doi: 10.1038/cr.2015.139

He, Y., Hara, H., and Núñez, G. (2016). Mechanism and regulation of nlrp3 inflammasome activation. Trends Biochem. Sci. 41, 1012-1021. doi: 10.1016/j. tibs.2016.09.002

Heijman, J., Algalarrondo, V., Voigt, N., Melka, J., Wehrens, X. H., Dobrev, D., et al. (2016). The value of basic research insights into atrial fibrillation mechanisms as a guide to therapeutic innovation: a critical analysis. Cardiovasc. Res. 109, 467-479. doi: 10.1093/cvr/cvv275

Heijman, J., Guichard, J. B., Dobrev, D., and Nattel, S. (2018). Translational challenges in atrial fibrillation. Circ. Res. 122, 752-773. doi: 10.1161/ CIRCRESAHA.117.311081

Heijman, J., Voigt, N., Nattel, S., and Dobrev, D. (2014). Cellular and molecular electrophysiology of atrial fibrillation initiation, maintenance, and progression. Circ. Res. 114, 1483-1499. doi: 10.1161/CIRCRESAHA.114.302226

Hoffman, H. M., and Wanderer, A. A. (2010). Inflammasome and IL-1 $\beta$-mediated disorders. Curr. Allergy Asthma Rep. 10, 229-235. doi: 10.1007/s11882-0100109-z 
Howley, B., and Fearnhead, H. O. (2008). Caspases as therapeutic targets. J. Cell. Mol. Med. 12, 1502-1516. doi: 10.1111/j.1582-4934.2008.00292.x

Jennings, R. B., Murry, C. E., Steenbergen, C. Jr., and Reimer, K. A. (1990). Development of cell injury in sustained acute ischemia. Circulation 82, II2-II12.

Jesus, A. A., and Goldbach-Mansky, R. (2014). IL-1 blockade in autoinflammatory syndromes. Annu. Rev. Med. 65, 223-244. doi: 10.1146/annurev-med-061512150641

Kanneganti, T. D. (2015). The inflammasome: firing up innate immunity. Immunol. Rev. Immunol. Rev. 265, 1-5. doi: 10.1111/imr.12297

Kaur, K., Dhingra, S., Slezak, J., Sharma, A. K., Bajaj, A., and Singal, P. K. (2009). Biology of TNF $\alpha$ and IL-10, and their imbalance in heart failure. Heart Fail. Rev. 14, 113-123. doi: 10.1007/s10741-008-9104-z

Kawaguchi, M., Takahashi, M., Hata, T., Kashima, Y., Usui, F., Morimoto, H., et al. (2011). Inflammasome activation of cardiac fibroblasts is essential for myocardial ischemia/reperfusion injury. Circulation 123, 594-604. doi: 10. 1161/CIRCULATIONAHA.110.982777

Lawrence, T. (2009). The nuclear factor NF-kappaB pathway in inflammation. Cold Spring Harb. Perspect. Biol. 1:a001651. doi: 10.1101/cshperspect.a001651

Li, P., Allen, H., Banerjee, S., Franklin, S., Herzog, L., Johnston, C., et al. (1995). Mice deficient in il-1-beta-converting enzyme are defective in production of mature il-1-beta and resistant to endotoxic-shock. Cell 80, 401-411. doi: 10. 1016/0092-8674(95)90490-5

Liu, X., Zhang, Z., Ruan, J., Pan, Y., Magupalli, V. G., Wu, H., et al. (2016). Inflammasome-activated gasdermin $\mathrm{D}$ causes pyroptosis by forming membrane pores. Nature 535, 153-158. doi: 10.1038/nature18629

Liu, Y., Lian, K., Zhang, L., Wang, R., Yi, F., Gao, C., et al. (2014). TXNIP mediates NLRP3 inflammasome activation in cardiac microvascular endothelial cells as a novel mechanism in myocardial ischemia/reperfusion injury. Basic Res. Cardiol. 109:415. doi: 10.1007/s00395-014-0415-z

Luan, Y., Li, S., Yu, B., Zhu, S., Li, S., Li, N., et al. (2010). Interleukin-18 among atrial fibrillation patients in the absence of structural heart disease. Europace 12, 1713-1718. doi: 10.1093/europace/euq321

Luo, B., Li, B., Wang, W., Liu, X., Xia, Y., Zhang, C., et al. (2014). NLRP3 gene silencing ameliorates diabetic cardiomyopathy in a type 2 diabetes rat model. PLoS One 9:e104771. doi: 10.1371/journal.pone.0104771

MacKenzie, S. H., Schipper, J. L., and Clark, A. C. (2010). The potential for caspases in drug discovery. Curr. Opin. Drug Discov. Devel. 13, 568-576.

Martinon, F., Burns, K., and Tschopp, J. (2002). The inflammasome: a molecular platform triggering activation of inflammatory caspases and processing of prolL-beta. Mol. Cell. 10, 417-426. doi: 10.1016/S1097-2765(02) 00599-3

Matzinger, P. (1994). Tolerance, danger, and the extended family. Annu. Rev. Immunol. 12, 991-1045. doi: 10.1146/annurev.iy.12.040194.005015

Medzhitov, R. (2008). Origin and physiological roles of inflammation. Nature 454, 428-435. doi: 10.1038/nature07201

Mezzaroma, E., Toldo, S., Farkas, D., Seropian, I. M., Van Tassell, B. W., Salloum, F. N., et al. (2011). The inflammasome promotes adverse cardiac remodeling following acute myocardial infarction in the mouse. Proc. Natl. Acad. Sci. U.S.A. 108, 19725-19730. doi: 10.1073/pnas.1108586108

Mitroulis, I., Skendros, P., and Ritis, K. (2010). Targeting IL-1beta in disease; the expanding role of NLRP3 inflammasome. Eur. J. Intern. Med. 21, 157-163. doi: 10.1016/j.ejim.2010.03.005

Nguyen, M. N., Kiriazis, H., Gao, X. M., and Du, X. J. (2017). Cardiac Fibrosis and Arrhythmogenesis. Compr. Physiol. 7, 1009-1049. doi: 10.1002/cphy.c160046

Opie, L. H., Commerford, P. J., Gersh, B. J., and Pfeffer, M. A. (2006). Controversies in ventricular remodelling. Lancet 367, 356-367. doi: 10.1016/S0140-6736(06) 68074-4

Pfeffer, M. A., and Braunwald, E. (1990). Ventricular remodeling after myocardial infarction. Experimental observations and clinical implications. Circulation 81, 1161-1172. doi: 10.1161/01.CIR.81.4.1161

Ridker, P. M., Everett, B. M., Thuren, T., MacFadyen, J. G., Chang, W. H., Ballantyne, C., et al. (2017). Antiinflammatory therapy with canakinumab for atherosclerotic disease. N. Engl. J. Med. 377, 1119-1131. doi: 10.1056/ NEJMoa1707914

Sandanger, O., Ranheim, T., Vinge, L. E., Bliksoen, M., Alfsnes, K., Finsen, A. V., et al. (2013). The NLRP3 inflammasome is up-regulated in cardiac fibroblasts and mediates myocardial ischaemia-reperfusion injury. Cardiovasc. Res. 99, 164-174. doi: 10.1093/cvr/cvt091
Sborgi, L., Ruhl, S., Mulvihill, E., Pipercevic, J., Heilig, R., Stahlberg, H., et al. (2016). GSDMD membrane pore formation constitutes the mechanism of pyroptotic cell death. EMBO J. 35, 1766-1778. doi: 10.15252/embj.201694696

Schroder, K., and Tschopp, J. (2010). The inflammasomes. Cell 140, 821-832. doi: 10.1016/j.cell.2010.01.040

Shao, W., Yeretssian, G., Doiron, K., Hussain, S. N., and Saleh, M. (2007). The caspase-1 digestome identifies the glycolysis pathway as a target during infection and septic shock. J. Biol. Chem. 282, 36321-36329. doi: 10.1074/jbc. M708182200

Shaw, J. E., Sicree, R. A., and Zimmet, P. Z. (2010). Global estimates of the prevalence of diabetes for 2010 and 2030. Diabetes Res. Clin. Pract. 87, 4-14. doi: 10.1016/j.diabres.2009.10.007

Shi, J., Zhao, Y., Wang, K., Shi, X., Wang, Y., Huang, H., et al. (2015). Cleavage of GSDMD by inflammatory caspases determines pyroptotic cell death. Nature 526, 660-665. doi: 10.1038/nature15514

Ting, J. P., Lovering, R. C., Alnemri, E. S., Bertin, J., Boss, J. M., Davis, B. K., et al. (2008). The NLR gene family: a standard nomenclature. Immunity 28, 285-287. doi: 10.1016/j.immuni.2008.02.005

Toldo, S., and Abbate, A. (2018). The NLRP3 inflammasome in acute myocardial infarction. Nat. Rev. Cardiol. 15, 203-214. doi: 10.1038/nrcardio.2017.161

Toldo, S., Marchetti, C., Mauro, A. G., Chojnacki, J., Mezzaroma, E., Carbone, S., et al. (2016). Inhibition of the NLRP3 inflammasome limits the inflammatory injury following myocardial ischemia-reperfusion in the mouse. Int. J. Cardiol. 209, 215-220. doi: 10.1016/j.ijcard.2016.02.043

Travers, J. G., Kamal, F. A., Robbins, J., Yutzey, K. E., and Blaxall, B. C. (2016). Cardiac fibrosis: the fibroblast awakens. Circ. Res. 118, 1021-1040. doi: 10.1161/ CIRCRESAHA.115.306565

Valle Raleigh, J., Mauro, A. G., Devarakonda, T., Marchetti, C., He, J., Kim, E., et al. (2017). Reperfusion therapy with recombinant human relaxin-2 (Serelaxin) attenuates myocardial infarct size and NLRP3 inflammasome following ischemia/reperfusion injury via eNOS-dependent mechanism. Cardiovasc. Res. 113, 609-619. doi: 10.1093/cvr/cvw246

Vandanmagsar, B., Youm, Y. H., Ravussin, A., Galgani, J. E., Stadler, K., Mynatt, R. L., et al. (2011). The NLRP3 inflammasome instigates obesity-induced inflammation and insulin resistance. Nat. Med. 17, 179-188. doi: 10.1038/nm. 2279

Wexler, R. K., Elton, T., Pleister, A., and Feldman, D. (2009). Cardiomyopathy: an overview. Am. Fam. Physician 79, 778-784.

Wilson, K. P., Black, J. A., Thomson, J. A., Kim, E. E., Griffith, J. P., Navia, M. A., et al. (1994). Structure and mechanism of interleukin-1 beta converting enzyme. Nature 28, 270-275. doi: 10.1038/370270a0

Yao, C., Veleva, T., Scott, L., Cao, S., Li, L., Chen, G., et al. (2018). Enhanced cardiomyocyte nlrp3 inflammasome signaling promotes atrial fibrillation. Circulation doi: 10.1161/CIRCULATIONAHA.118.035202 [Epub ahead of print].

Yin, Y., Yan, Y., Jiang, X., Mai, J., Chen, N. C., Wang, H., et al. (2009). Inflammasomes are differentially expressed in cardiovascular and other tissues. Int. J. Immunopathol. Pharmacol. 22, 311-322. doi: 10.1177/ 039463200902200208

Zhang, F., Wen, Y., and Guo, X. (2014). CRISPR/Cas9 for genome editing: progress, implications and challenges. Hum. Mol. Genet. 23, R40-R46. doi: 10.1093/hmg/ddu125

Zu, Y., Wan, L. J., Cui, S. Y., Gong, Y. P., and Li, C. L. (2015). The mitochondrial $\mathrm{Na}+/ \mathrm{Ca} 2+$ exchanger may reduce high glucose-induced oxidative stress and nucleotide-binding oligomerization domain receptor 3 inflammasome activation in endothelial cells. J. Geriatr. Cardiol. 12, 270-278.

Conflict of Interest Statement: The authors declare that the research was conducted in the absence of any commercial or financial relationships that could be construed as a potential conflict of interest.

Copyright (C) 2018 Chen, Chelu, Dobrev and Li. This is an open-access article distributed under the terms of the Creative Commons Attribution License (CC BY). The use, distribution or reproduction in other forums is permitted, provided the original author(s) and the copyright owner(s) are credited and that the original publication in this journal is cited, in accordance with accepted academic practice. No use, distribution or reproduction is permitted which does not comply with these terms. 